\section{Migräne: Weniger Attacken und Akutmedikationen mit Erenumab}

Goadsby PJ et al. A Controlled Trial of Erenumab for Episodic Migraine. N Engl J Med 2017; 377: 2123-2132.

Bisherige Studien mit Antikörpern wiesen bereits daraufhin, dass die Beeinflussung des Signalwegs des „calcitonin gene-related peptide“" (CGRP) ein wichtiges Ziel der Migränetherapie sein könnte. Mit einer Phase-3-Studie belegen Goadsby et al. bei Patienten mit episodischer Migräne die Wirksamkeit und Sicherheit des monoklonalen Antikörpers Erenumab, der selektiv an den CGRP-Rezeptor bindet.

An der „Study to Evaluate the Efficacy and Safety of Erenumab in Migraine Prevention“ (STRIVE) konnten Migräniker teilnehmen, bei denen 3 Monate vor dem Screening und in einer 4-wöchigen Ausgangsphase $\geq 4$ und $<15$ Migränetage pro Monat und <15 Kopfschmerztage pro Monat auftraten. Die randomisierte, doppelblinde, placebokontrollierte Parallelgruppen-Studie wurde in 121 Kliniken Nordamerikas, Europas und der Türkei durchgeführt. Am Schluss der Ausgangsphase fand die Randomisierung auf monatliche subkutane Injektionen von 70 mg bzw. 140 mg Erenumab oder Placebo am Tag 1 und zu den Wochen 4, 8, 12, 16 und 20 statt. Veränderungen der mittleren Anzahl an Migränetagen vom Ausgangswert bis zu den Monaten 4-6 bildeten den primären Endpunkt. Das sekundäre Studienziel war definiert als $\geq 50$-prozentige Reduktion der durchschnittlichen monatlichen Migränetage, Veränderungen der Anzahl an Akutmedikationstagen und im „Migraine Physical Function Impact Diary“ (MPFID).

\section{Erenumab punktet gegenüber Placebo}

Von den insgesamt 955 Teilnehmern erhielten 317 Patienten $70 \mathrm{mg}$ des Antikörpers, 319 die Dosierung mit $140 \mathrm{mg}$ und 319 Placebo. Die 6-monatige DoppelblindPhase beendeten 89,8\% der Patienten. In der Ausgangsphase hatten alle Studienteilnehmer durchschnittlich 8,3 Migränetage pro Monat, $58,8 \%$ benötigten
Akutmedikationen und 2,8\% nahmen Medikamente zur Migräneprävention ein. Die Migränetage gingen in der $70 \mathrm{mg}$-Verumgruppe im Mittel um 3,2 Tage und unter 140 mg Verum um 3,7 Tage zurück; die Reduktion unter Placebo erreichte 1,8 Tage.

Während in der Placebogruppe 26,6\% der Patienten von einer $\geq 50$-prozentigen Reduktion der durchschnittlichen monatlichen Migränetage profitierten, waren es unter $70 \mathrm{mg}$ Erenumab 43,3\% und unter $140 \mathrm{mg} 50 \%$. Im Placebovergleich war die Chance auf eine $\geq 50$ prozentige Reduktion der durchschnittlichen monatlichen Migränetage zu den Monaten 4-6 unter Verum 2,1-mal (70 mg) bzw. 2,8-mal (140 mg) größer. Die Anzahl der Tage, an denen Akutmedikationen eingenommen wurden, reduzierte sich in den Verumgruppen um 1,1 Tage $(70 \mathrm{mg})$ bzw. 1,6 Tage (140 $\mathrm{mg}$ ) und unter Placebo um 0,2 Tage. Die Bewertung körperlicher Beeinträchtigungen verbesserte sich um 4,2 Punkte und 4,8 Punkte (70 mg und $140 \mathrm{mg}$ Erenumab), unter Placebo dagegen um 2,4 Punkte. In Bezug auf Alltagsaktivitäten verbesserte sich die Situation um 5,5 Punkte und 5,9 Punkte (70 mg und $140 \mathrm{mg}$ Erenumab), in der Placebogruppe um 3,3 Punkte. Unerwünschte Ereignisse jeder Ausprägung lagen in den beiden Verumgruppen auf Placeboniveau.

FAZIT

Bei episodischer Migräne führte Erenumab in dieser Studie gegenüber Placebo zu deutlichen klinischen Vorteilen, die begleitet waren von einem reduzierten Bedarf an Akutmedikation sowie Verbesserungen bei körperlichen Beeinträchtigungen und Alltagsaktivitäten.

Matthias Manych, Berlin 